\title{
SYNTHESIS OF TETRASUBSTITUTD IMIDAZOLES CONTAINING INDOLE AND THEIR ANTIUREASE AND ANTIOXIDANT ACTIVITIES
}

\author{
SADIA NAUREEN ${ }^{\prime *}$, FATIMA IJAZ ${ }^{1}$, MUNAWAR ALI MUNAWAR ${ }^{1}$, NADIA ASIF ${ }^{1}$, FARYAL CHAUDHR $Y^{1}$, \\ MUHAMMAD ASHRAFF ${ }^{2,3}$ AND MISBAHUL AIN KHAN ${ }^{1,2}$
}

\author{
'Institute of Chemistry, University of the Punjab, Quaid-e-Azam campus, Lahore, Pakistan. \\ ${ }^{2}$ Department of Chemistry, The Islamia University of Bahawalpur, Bahawalpur - 63100, Pakistan. \\ ${ }^{3}$ Department of Biochemistry and Biotechnology, The Islamia University of Bahawalpur, Bahawalpur - 63100, Pakistan.
}

\begin{abstract}
Indole-based tetraarylimidazoles were prepared by multicomponent reaction of substituted 2-arylindole-3-carbaldehydes, benzil, substituted anilines and ammonium acetate in acetic acid. The new compounds were evaluated for their antiurease and antioxidant activity. The synthesized compounds exhibited potent antiurease activity.
\end{abstract}

Keywords: Imidazole; Indole; antitumor; ammonium acetate.

\section{INTRODUCTION}

Indole skeleton is a building block of a number of natural and synthetic products found in plants and animals or synthesized in the laboratory. Indole derivatives fascinated chemists because of their significant range of pharmacological activities such as anticonvulsant ${ }^{1}$, antitumor ${ }^{2}$, antiinflammatory ${ }^{3}$, antimicrobial ${ }^{4}$ and antifungal $^{5}$.

Further, it has been reported that substitution of different heterocyclic or aromatic moieties at 2 or 3-position of indole nucleus modulates various biological activities of such substituted indole derivatives ${ }^{3}$. The incorporation of the imidazole ring system is a synthetic approach in drug invention. The therapeutic properties of imidazole entity are a major source of attention for medicinal chemists. The imidazole ring system has received much attention because of its anti-inflammatory ${ }^{6}$, antimicrobial ${ }^{7}$, anticonvulsant ${ }^{8}$, anticancer ${ }^{9}$, analgesic $^{10}$, fungicidal ${ }^{11}$ and antithrombotic activities ${ }^{12}$. Imidazole derivatives have also been reported in compounds which are used for electronics, photography and as fire retardants ${ }^{13}$.

It is therefore thought worthwhile to synthesize some new indole derivatives by incorporating imidazole nucleus with the hope that it may exhibit still other interesting biological activities. We have recently reported the synthesis of a number of indole based triarylimidazoles ${ }^{14}$ and tetraarylimidazoles ${ }^{15}$ which possess excellent $\alpha$-glucosidase inhibitory and antiurease activity respectively. In view of such importance of these imidazole and indole ring compounds, we would like to communicate our continuing efforts in preparing still more tetraarylimidazoles containing a 2-indol-3-yl substituents and their screening as antiurease and antioxidant agents.

Urease is an enzyme that catalyzes the hydrolysis of urea into ammonia and carbon dioxide. It is found in various types of bacteria, fungi and plants. It is the main source of pathology provoked by Helicobacter pylori (Gram negative bacterium) is found in the stomach and play an important part in the development of gastric and duodenal ulcers ${ }^{16}$. Urease has different functions and antiurease agents have received special interest over the past few years. Urease inhibitors are classified into two major classes of organic compounds such as Thiourea ${ }^{17}$, Omeprazole ${ }^{18}$ (imidazoles) (Fig.1), coumarins ${ }^{19}$, quinines and triazoles ${ }^{20}$ and as metal organic such as bishydrazone derivatives and its copper (II) complexes ${ }^{21}$ (Fig.2) has been reported.<smiles>COc1ccc2[nH]c(S(=O)Cc3ncc(C)c(OC)c3C)nc2c1</smiles>



Fig.2 Structure of $\mathrm{Cu}(\mathrm{II})$ complexes with bishydrazones.

Antioxidant acts as a weapon to shield the body against oxidative stress. Antioxidants reduce degenerative diseases such as cancers and cardiovascular diseases by trapping free radicals. Certain imidazole derivatives ${ }^{22}$ (Fig.3), indole derivatives ${ }^{23}$ (Fig.4) and imidazolopyridinyl indoles ${ }^{24}$ (Fig.5) have been found to possess antioxidant activity.

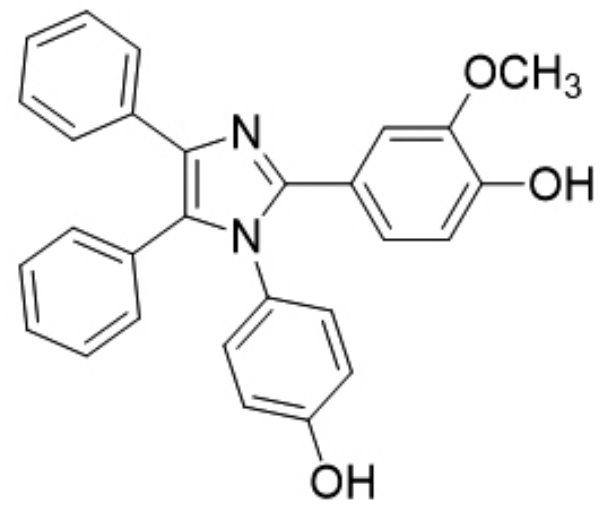

Fig.3 4-(1-(4-hydroxyphenyl) -4,5-diphenyl-1-1H-X=H,F R=Thiazolyl imidazole-2-yl)2-yl)-2-methoxyphenol.

Fig.1 Omeprazole 

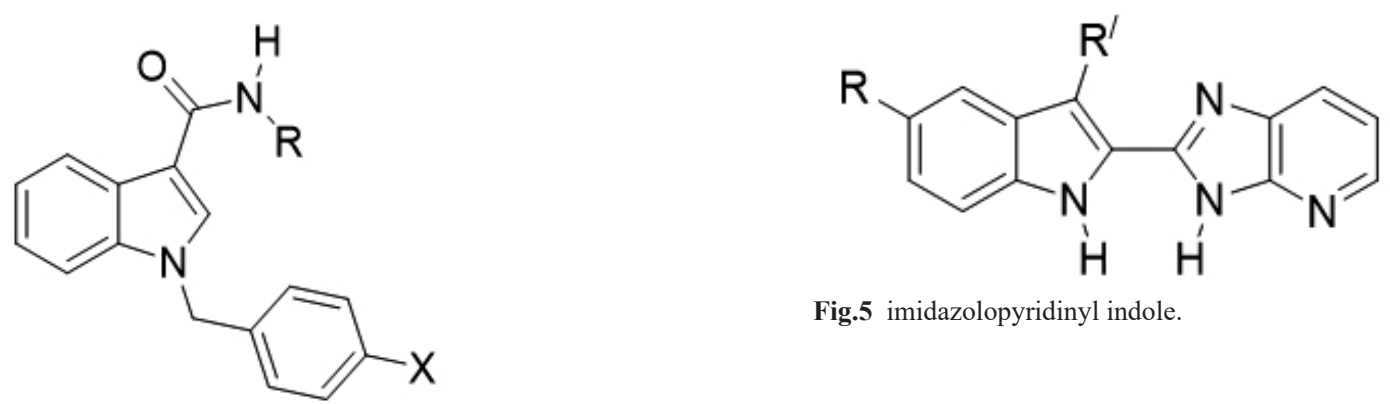

Fig.5 imidazolopyridinyl indole.

Fig.4 Indole-3-carboxamide $\mathrm{R}=\mathrm{Cl}, \mathrm{Br}, \mathrm{CH}_{3}, \mathrm{OCH}_{3} \mathrm{R}=\mathrm{Ph}$

\section{RESULTS AND DISCUSSIONS}

Chemistry

Substituted 2-arylindoles were prepared by the method of Fischer indole synthesis ${ }^{25}$ which were subsequently formylated using Vilsmeir-Haack ${ }^{26}$ reaction The 2-arylindole-3-carbaldehydes, ammonium acetate, benzil and substituted anilines were the reactants used in this MCR (Multi component reaction) to provide a new series of tetraarylimidazoles (Scheme 1,4a-4j).

The structures of these compounds were confirmed on the basis of spectral data. In their HRMS, the molecular ion peaks were found to correspond to their expected values. The ${ }^{1} \mathrm{HNMR}$ spectra showed a singlet for $-\mathrm{NH}$ of indole in the range of $\delta 11-12$. The compounds substituted with $-\mathrm{CH}_{3}$ and $-\mathrm{OCH},(\mathbf{4 a}, \mathbf{4 d}$, $\mathbf{4 f}, \mathbf{4 g}, \mathbf{5 h}, \mathbf{5 i}, \mathbf{4 j}$ ) showed upfield singlet in the range of $\delta 2-4$. The splitting patterns of remaining protons of spectra were as expected, and were according to the substituents. The ${ }^{13} \mathrm{CNMR}$ spectra and FTIR were also consistent with the structures.<smiles>[R][R]1ccc(-c2[nH]c3ccc([R])cc3c2C=O)cc1</smiles>

substituted aldehydes<smiles>O=C(C(=O)c1ccccc1)c1ccccc1</smiles>

benzil<smiles>[R]c1ccc(N)cc1</smiles>

substituted anilines



$(4 a-4 j)$

${ }^{1} \mathrm{R}=\mathrm{H}, \mathrm{Br}$

${ }^{2} \mathrm{R}=\mathrm{H}, \mathrm{Cl}, \mathrm{CH}_{3}, \mathrm{Br} \quad{ }^{3} \mathrm{R}=\mathrm{H}, \mathrm{Br}, \mathrm{F}, \mathrm{Cl}, \mathrm{CH}_{3}, \mathrm{OCH}_{3}$

Scheme-1 Synthetic route for the synthesis of compounds $(\mathbf{4 a}-\mathbf{4 j})$.

Table 1: Antiurease and antioxidant activity by indole-imidazole derivatives

\begin{tabular}{|c|c|c|c|c|c|c|c|c|}
\hline \multirow{2}{*}{ Sr. No. } & \multirow{2}{*}{ Sample code } & \multirow{2}{*}{${ }^{1} \mathrm{R}$} & \multirow{2}{*}{${ }^{2} \mathrm{R}$} & \multirow{2}{*}{${ }^{3} \mathrm{R}$} & \multicolumn{2}{|c|}{ Antiurease } & \multicolumn{2}{|c|}{ Antioxidant } \\
\hline & & & & & Inhibition $(\%)$ at $0.5 \mathrm{mM}$ & $\mathrm{IC}_{50}(\mu \mathrm{M})$ & Inhibition (\%) at $0.5 \mathrm{mM}$ & $\mathrm{IC}_{50}(\mu \mathrm{M})$ \\
\hline 1 & $4 a$ & $\mathrm{H}$ & $\mathrm{Cl}$ & $\mathrm{CH}_{3}$ & $76.44 \pm 0.8$ & $47.53 \pm \mathbf{0 . 1 2}$ & $62.58 \pm 0.7$ & $175.26 \pm 1.24$ \\
\hline 2 & $4 b$ & $\mathrm{H}$ & $\mathrm{Cl}$ & $\mathrm{Br}$ & $76.54 \pm 0.8$ & $47.64 \pm \mathbf{0 . 1 5}$ & $71.74 \pm 0.2$ & $146.27 \pm 1.09$ \\
\hline 3 & $4 c$ & $\mathrm{Br}$ & $\mathrm{H}$ & $\mathrm{F}$ & $92.58 \pm 0.3$ & $11.35 \pm 0.07$ & $71.87 \pm 0.5$ & $181.26 \pm 1.1$ \\
\hline 4 & 4d & $\mathrm{H}$ & $\mathrm{Br}$ & $\mathrm{CH}_{3}$ & $87.44 \pm 0.8$ & $9.95 \pm \mathbf{0 . 0 5}$ & $90.39 \pm 0.5$ & $148.26 \pm 1.2$ \\
\hline 6 & $4 \mathrm{f}$ & $\mathrm{H}$ & $\mathrm{CH}_{3}$ & $\mathrm{H}$ & $85.77 \pm 0.6$ & $4.85 \pm 0.07$ & $67.61 \pm 0.3$ & $162.27 \pm 1.2$ \\
\hline 7 & $4 g$ & $\mathrm{H}$ & $\mathrm{CH} 3$ & $\mathrm{CH}_{3}$ & $86.73 \pm 0.7$ & $0.16 \pm 0.04$ & $44.21 \pm 0.7$ & - \\
\hline 8 & $4 h$ & $\mathrm{H}$ & $\mathrm{CH}_{3}$ & $\mathrm{Br}$ & $87.38 \pm 0.46$ & $1.45 \pm 0.05$ & $7.11 \pm 0.2$ & - \\
\hline 9 & $4 i$ & $\mathrm{H}$ & $\mathrm{CH}_{3}$ & F & $89.73 \pm 0.9$ & $4.31 \pm 0.02$ & $18.91 \pm 0.6$ & - \\
\hline \multirow[t]{2}{*}{10} & $4 j$ & $\mathrm{H}$ & $\mathrm{CH}_{3}$ & $\mathrm{OCH}_{3}$ & $87.63 \pm 0.9$ & $0.12 \pm 0.02$ & $23.03 \pm 0.5$ & - \\
\hline & Quercetin & & & & - & & $93.21 \pm 0.9$ & $16.96 \pm 0.1$ \\
\hline
\end{tabular}


Antiurease activity.

The decreasing order of activity of these compounds is $4 j>4 g>4 h>4 i>4 f>4 d>4 c>4 e>4 a>4 b$

Most potent active compound (4j) has substituent $\mathrm{R}=\mathrm{H}, \mathrm{R}=\mathrm{CH}_{3}$ and $\mathrm{R}_{3}=\mathrm{OCH}_{3}$; Compounds substituted with $\mathrm{R}_{1}=\mathrm{H} . \mathrm{R}_{2}=\mathrm{Cl}$ and $\mathrm{R}_{3}=\mathrm{CH}_{3}$ and $\mathrm{R}_{1}=\mathrm{H}$, $\mathrm{R}_{2}=\mathrm{Cl}$ and $\mathrm{R}_{3}=\mathrm{Br}(\mathbf{4 a}, \mathbf{4 b})$ displayed lesser inhibition towards this enzyme.

Outstanding activity was exhibited by compound $\mathbf{4 j}$. It was found 177.78 times more potent as compared to the standard and the compound $\mathbf{4 g}\left(\mathrm{IC}_{50=}\right.$ $0.16)$ disubstituted with $-\mathrm{CH}_{3}$ were 132.8 times more active than the reference. The compound $\mathbf{4 h}\left(\mathrm{IC}_{50=} 1.45\right)$ substituted with a $\mathrm{CH}_{3}$ and a $\mathrm{Br}$ still 14.65 times more active than standard.

The compounds $4 \mathbf{i}\left(\mathrm{IC}_{50}\right.$ 4.31) substituted with a $\mathrm{CH}_{3}$ and a $\mathrm{F}$ and compound $4 \mathbf{f}\left(\mathrm{IC}_{50}=4.85\right)$ substituted with the $\mathrm{CH}_{3}$ have been found 4.93 and 4.38 times, respectively more potent than the standard, while compounds $4 \mathbf{d}$ ( $\mathrm{IC}_{50=}$ 9.95) substituted with $\mathrm{Br}$ and $\mathrm{CH}_{3}$, compound $\mathbf{4 c}\left(\mathrm{IC}_{50=} 11.35\right)$ substituted with $\mathrm{Br}$ and $\mathrm{F}$ and $4 \mathrm{e}\left(\mathrm{IC}_{50=} 20.25\right)$ substituted with $\mathrm{Cl}$ and $\mathrm{Br}$ were $2.13,1.87$ and 1.04 times respectively more active than the standard.

The compound $4 \mathbf{a}$ ( $\mathrm{IC}_{50} 47.53$ ) substituted with $\mathrm{CH}_{3}$ and $\mathrm{Cl}$ and compound 4b $\left(\mathrm{IC}_{50}=47.64\right)$ substituted with $\mathrm{Cl}$ and $\mathrm{Br}$ were found 2.23 and 2.24 times respectively less active as compared to the standard.

It seems that in general compounds containing electron donating groups $\left(\mathrm{CH}_{3}\right.$ and a halogen $)$ in general are potent candidates as more active antiureases. Antioxidant Activity

The compounds of this series were also tested for their antioxidant activities as compared to Quercetin against DPPH. The compounds $\mathbf{4 a}, \mathbf{4 b}, \mathbf{4} \mathbf{c}, \mathbf{4 d}, \mathbf{4 f}$ were found active with decreasing order of this activity being $\mathbf{4 b}>\mathbf{4 d}>\mathbf{4 f}>\mathbf{4 a}>\mathbf{4 c}$ .These compounds exhibited weak antioxidant activities and are listed in table 1. Among tested compounds the compound 4d substituted with $\mathrm{CH}_{3}$ and $\mathrm{Br}$ showed the highest antioxidant inhibition of $90.3 \pm 0.57 \%$ at $0.5 \mathrm{mM}$ and $\mathrm{IC}_{50}$ $148.2 \pm 1 \mathrm{mM}$ and compound $\mathbf{4 b}$ substituted with $\mathrm{Cl}$ and $\mathrm{Br}$ with inhibition of $71.7 \pm 0.27 \%$ and IC50 $146.27 \pm 1.09 \mathrm{mM}$. This data suggest that such compounds are weak antioxidant agents.

Conclusions

A series of tetrasubstituted imidazoles containing a 2-arylindole substituent has been prepared by one-pot four component condensation reaction. The new compounds showed significant antiurease activity as compared to standard inhibitor, thiourea. However, low inhibition profiles were observed for the antioxidant activity.

\section{MATERIALS AND METHODS}

The chemicals and solvent used in this experimental work were of analytical grade and were purchased from Merck, Fluka and Aldrich and were used as such. Melting points were determined on a Gallen Kamp melting point apparatus in open capillary tubes and are uncorrected. High resolution mass spectra were recorded on Waters LCT Premier XE TOF-MS. Analytical TLC was performed on DCAlufolien Silica Gel $60 \mathrm{~F}_{254}$ Merck. UV lamp of short and long wavelength (model UVGL-25 minor light multiband UV-254/366) was used to visualize TLC plates. IR spectra were recorded on Perkin Elmer Spectrum BX FT-IR. ${ }^{1} \mathrm{H}$ spectra were recorded at $500 \mathrm{MHZ}$ and ${ }^{13} \mathrm{C}$ NMR spectra were recorded at 126 MHZ on a Brucker Avance AV11500B spectrometer.

General Procedure for the synthesis of Tetrasubstituted imidazoles containing a substituted indole $(4 a-4 j)$

A mixture of benzil (1.0 equiv), a substituted 2-phenylindole 3 -carbaldehyde (1.0 equiv) an aromatic amine (1.0 equiv) and ammonium acetate (4.0 equiv) in acetic acid was heated at reflux for 5-6 hours ${ }^{15}$. After the completion of the reaction (monitored by TLC) and cooling to room temperature, the reaction mixture was poured into cold water. The precipitated product was filtered, washed with excess of water and recrystallized with EtOH to obtain pure $(\mathbf{4 a - 4 j})$.

The following compounds were prepared from this general method:

2-(p-Chlorophenyl)-3-(4,5-diphenyl-1-(p-tolyl)-1H-imidazol-2-yl)-1Hindole (4a)

Yield: $57 \%$ as a white solid.

mp: $265-270{ }^{\circ} \mathrm{C}$

IR (neat): $3063,2356,1514,1389,1366,1095,1020 \mathrm{~cm}^{-1}$

HRMS $\left(\mathrm{ES}^{+}\right)$calcd. for $\mathrm{C}_{36} \mathrm{H}_{27} \mathrm{ClN}_{3}[\mathrm{M}+\mathrm{H}]^{+}$536.1894 Found: 536.1882; $\mathrm{m} / \mathrm{z}(\%): 536.1882\left([\mathrm{M}+\mathrm{H}]^{+}, 100 \%\right), 538.1915(30 \%)$

${ }^{1} \mathrm{H}$ NMR (500 MHz, DMSO- $\left.d_{6}\right) \delta: 11.64(\mathrm{~s}, 1 \mathrm{H}), 7.60$ - $7.44(\mathrm{~m}, 3 \mathrm{H}), 7.45$ $(\mathrm{d}, J=8.5 \mathrm{~Hz}, 2 \mathrm{H}), 7.39(\mathrm{~d}, J=7.9 \mathrm{~Hz}, 1 \mathrm{H}), 7.32-7.20(\mathrm{~m}, 9 \mathrm{H}), 7.20-7.12$ (m, 2H), 7.03-7.08 (m, 1H), 6.69 (d, $J=8.0 \mathrm{~Hz}, 2 \mathrm{H}), 6.46$ (d, $J=6.8 \mathrm{~Hz}, 2 \mathrm{H})$, $2.05(\mathrm{~s}, 3 \mathrm{H})$.

${ }^{13} \mathrm{C}$ NMR $(126 \mathrm{MHz}$, DMSO- $d$ ) $\delta: 142.69,139.59,139.33,139.13$, $137.59,137.36,136.58,136.36,135.35,135.19,133.90,133.11,131.68$, $131.48,131.29,130.56,130.32,130.03,129.65,129.18,129.12,129.08$, $128.85,128.79,128.74,127.66,127.58,126.88,123.07,120.69,120.08$, $113.85,112.06,103.92,21.14$

3-(1-(p-Bromophenyl)-4,5-diphenyl-1H-imidazol-2-yl)-2-( $p$ chlorophenyl)- $1 H$-indole $(4 \mathrm{~b})$

Yield: $68 \%$ as a white solid.

mp: $265{ }^{\circ} \mathrm{C}$;

IR (neat) 3065, 2359, 1490, 1386, 1224, 1070, $1015 \mathrm{~cm}^{-1}$;

HRMS $\left(\mathrm{ES}^{+}\right)$calcd. for $\mathrm{C}_{35} \mathrm{H}_{24} \mathrm{BrClN}_{3}[\mathrm{M}+\mathrm{H}]^{+} 600.0842$ Found: 600.0836; $\mathrm{m} / \mathrm{z}(\%): 600.0836\left([\mathrm{M}+\mathrm{H}]^{+}, 80 \%\right), 602.0800(100 \%)$

${ }^{1} \mathrm{H}$ NMR $\left(500 \mathrm{MHz}, \mathrm{DMSO}-d_{6}\right) \delta: 11.71(\mathrm{~s}, 1 \mathrm{H}), 7.62(\mathrm{~d}, J=7.9 \mathrm{~Hz}, 1 \mathrm{H})$, $7.54(\mathrm{~d}, J=7.3 \mathrm{~Hz}, 2 \mathrm{H}), 7.45-7.39(\mathrm{~m}, 3 \mathrm{H}), 7.35-7.17(\mathrm{~m}, 12 \mathrm{H}), 7.11-7.06$ (m, 2H), $6.47(\mathrm{~d}, J=6.2 \mathrm{~Hz}, 2 \mathrm{H})$.

${ }^{13} \mathrm{C}$ NMR (126 MHz, DMSO- $\left.d_{6}\right) \delta: 142.19,136.78,136.56,135.55$, $133.28,131.68,131.53,130.36,129.86,129.25,129.05,128.70,127.73$, $126.93,123.15,120.72,120.07,112.12,111.97,109.55,105.09$.

5-Bromo-3-(1-(p-fluorophenyl)-4,5-diphenyl-1' $H$-imidazol-2-yl)-2phenyl-1H-indole $(4 \mathrm{c})$

Yield: $83 \%$ as a white solid.

mp: $>300{ }^{\circ} \mathrm{C}$;

IR (neat): $3178,1602,1508,1313,1219,1152,1046 \mathrm{~cm}^{-1}$;

HRMS (ES ${ }^{+}$calcd. for $\mathrm{C}_{35} \mathrm{H}_{24} \mathrm{BrFN}_{3}[\mathrm{M}+\mathrm{H}]^{+} 584.1138$ Found: 584.1136; $\mathrm{m} / \mathrm{z}(\%): 584.1136\left([\mathrm{M}+\mathrm{H}]^{+}, 100 \%\right), 586.1129(100 \%)$

${ }^{1} \mathrm{H}$ NMR $(500 \mathrm{MHz}$, DMSO-d $) \delta: 11.83(\mathrm{~s}, 1 \mathrm{H}), 7.78(\mathrm{~s}, 1 \mathrm{H}), 7.55(\mathrm{~d}, J=$ $8.1 \mathrm{~Hz}, 2 \mathrm{H}), 7.39-7.18(\mathrm{~m}, 15 \mathrm{H}), 6.72-6.68(\mathrm{~m}, 2 \mathrm{H}), 6.48(\mathrm{~d}, J=6.7 \mathrm{~Hz}, 2 \mathrm{H})$

${ }^{13} \mathrm{C}$ NMR (126 MHz, DMSO- $\left.d_{6}\right) \delta: 160.15,142.31,139.44,137.71$, $135.21,135.18,132.63,131.93,131.65,131.28,131.23,130.46,129.82$ $129.74,129.26,129.17,128.97,128.93,128.81,127.86,127.00,126.93$, $125.35,122.15,115.44,115.26,114.14,113.20,102.68$.

2-(p-Bromophenyl)-3-(4,5-diphenyl-1-(p-tolyl)-1H-imidazol-2-yl)- $1 H$ indole (4d)

Yield: $52 \%$ as a white solid.

mp: $278^{\circ} \mathrm{C}$;

HRMS $\left(\mathrm{ES}^{+}\right.$) calcd. for $\mathrm{C}_{36} \mathrm{H}_{27} \mathrm{BrN}_{3}[\mathrm{M}+\mathrm{H}]^{+} 580.1388$ Found: 580.1380 ; $\mathrm{m} / \mathrm{z}(\%): 580.1\left([\mathrm{M}+\mathrm{H}]^{+} 100 \%\right) 582.1(100 \%)$

IR (neat): $3056,2360,1514,1074,846 \mathrm{~cm}^{-1}$;

${ }^{1} \mathrm{H}$ NMR (500 MHz, DMSO- $\left.d_{6}\right) \delta: 11.66(\mathrm{~s}, 1 \mathrm{H}), 7.63-7.50(\mathrm{~m}, 5 \mathrm{H}), 7.39$ $(\mathrm{d}, J=7.9 \mathrm{~Hz}, 1 \mathrm{H}), 7.32-7.20(\mathrm{~m}, 9 \mathrm{H}), 7.19-7.13(\mathrm{~m}, 2 \mathrm{H}), 7.09-7.02(\mathrm{~m}$, $1 \mathrm{H}), 6.68(\mathrm{~d}, J=7.9 \mathrm{~Hz}, 2 \mathrm{H}), 6.48(\mathrm{~d}, J=6.8 \mathrm{~Hz}, 2 \mathrm{H}), 2.04(\mathrm{~s}, 3 \mathrm{H})$

${ }^{13} \mathrm{C}$ NMR (126 MHz, DMSO- $\left.d_{6}\right) \delta: 142.45,142.23,137.72,137.49$, $136.58,135.16,132.13,131.99,131.69,131.10,130.59,130.29,129.45$ $129.18,129.12,128.79,128.74,127.66,127.59,127.00,126.89,124.50$, $122.73,121.62,120.66,114.36,112.06,103.93,21.00$

2-(p-Bromophenyl)-3-(1-(p-chlorophenyl)-4,5-diphenyl-1 $H$-imidazol2 -yl)-1H-indole $(4 \mathrm{e})$

Yield: $86 \%$ as a white solid.

mp: $276{ }^{\circ} \mathrm{C}$;

IR (neat): 3068(C-H), 2364, 1602, 1494, 1388, 1240, 1094, $1017 \mathrm{~cm}^{-1}$,

HRMS (ES $)$ calcd. for $\mathrm{C}_{35} \mathrm{H}_{24} \mathrm{BrClN}_{3}[\mathrm{M}+\mathrm{H}]^{+} 600.0842$ Found: 600.0827 ; $\mathrm{m} / \mathrm{z}(\%): 600.0827\left([\mathrm{M}+\mathrm{H}]^{+} 80 \%\right), 602.0825(100 \%)$

${ }^{1} \mathrm{H}$ NMR $\left(500 \mathrm{MHz}, \mathrm{DMSO}-d_{6}\right) \delta: 11.71(\mathrm{~s}, 1 \mathrm{H}), 7.65-7.52(\mathrm{~m}, 5 \mathrm{H}), 7.41$ $(\mathrm{d}, J=8.2 \mathrm{~Hz}, 1 \mathrm{H}), 7.36-7.04(\mathrm{~m}, 12 \mathrm{H}), 6.96(\mathrm{~d}, J=8.3 \mathrm{~Hz}, 2 \mathrm{H}), 6.55(\mathrm{~d}, J$ $=6.7 \mathrm{~Hz}, 2 \mathrm{H})$.

${ }^{13} \mathrm{C}$ NMR (75 MHz, DMSO-d $) \delta 141.93,137.23,136.01,135.85,134.63$, $134.50,131.93,131.53,131.32,131.07,130.90,130.47,129.93,129.58$, $128.92,128.89,128.79,128.59,128.52,128.41,128.13,127.93,127.31$, $127.02,126.39,126.30,122.52,121.23,121.21,120.10,119.99,119.54$, $111.50,111.40,102.76$ 
2-(p-Tolyl)-3-(1,4,5-triphenyl- $1 H$-imidazol-2-yl)-1 $H$-indole (4f) Yield: $54 \%$ as a yellow solid mp: $260{ }^{\circ} \mathrm{C}$;

IR (neat): 3068, 1598, 1496, 1392, 1089, $1050 \mathrm{~cm}^{-1}$;

HRMS $\left(\mathrm{ES}^{+}\right)$calcd. for $\mathrm{C}_{36} \mathrm{H}_{28} \mathrm{~N}_{3}[\mathrm{M}+\mathrm{H}]^{+} 502.2283$ Found: 502.2269

${ }^{1} \mathrm{H}$ NMR $(500 \mathrm{MHz}, \mathrm{DMSO}-d) \delta: 11.48(\mathrm{~s}, 1 \mathrm{H}), 7.57-7.49(\mathrm{~m}, 3 \mathrm{H}), 7.36$ $(\mathrm{d}, J=8.1 \mathrm{~Hz}, 1 \mathrm{H}), 7.30-7.09(\mathrm{~m}, 13 \mathrm{H}), 7.04-6.85(\mathrm{~m}, 4 \mathrm{H}), 6.56(\mathrm{~d}, J=7.0$ $\mathrm{Hz}, 2 \mathrm{H}), 2.30$ (s, 3H).

${ }^{13} \mathrm{C}$ NMR (126 MHz, DMSO- $\left.d_{6}\right) \delta: 143.15,138.20,137.90,137.65$, $136.58,136.29,135.45,131.60,131.42,130.35,129.82,129.58,129.08$ $128.76,128.50,127.85,127.50,127.00,126.86,122.51,120.40,119.76$ $111.91,103.02,21.39$. (4g)

3-(4,5-Diphenyl-1-(p-tolyl)-1H-imidazol-2-yl)-2-( $p$-tolyl)-1H-indole

\section{Yield: $68 \%$ as a yellow solid.}

mp: $270{ }^{\circ} \mathrm{C}$;

IR (neat): 3055, 1600, 1514, 1330, 1146, $1050 \mathrm{~cm}^{-1}$;

HRMS (ES ${ }^{+}$calcd. for $\mathrm{C}_{37} \mathrm{H}_{30} \mathrm{~N}_{3}[\mathrm{M}+\mathrm{H}]^{+} 516.2440$ Found: 516.2436 ;

${ }^{1} \mathrm{H}$ NMR (500 MHz, DMSO-d $\left.d_{6}\right) \delta: 11.53(\mathrm{~s}, 1 \mathrm{H}), 7.56-7.47(\mathrm{~m}, 3 \mathrm{H}), 7.37$ $(\mathrm{d}, J=8.0 \mathrm{~Hz}, 1 \mathrm{H}), 7.32-6.99(\mathrm{~m}, 14 \mathrm{H}), 6.68(\mathrm{~d}, J=7.9 \mathrm{~Hz}, 2 \mathrm{H}), 6.49(\mathrm{~d}, J=$ $6.1 \mathrm{~Hz}, 2 \mathrm{H}), 2.31(\mathrm{~s}, 3 \mathrm{H}), 2.03(\mathrm{~s}, 3 \mathrm{H})$.

${ }^{13} \mathrm{C}$ NMR (126 MHz, DMSO- $\left.d_{6}\right) \delta: 143.13,138.07,137.92,137.47$, $137.29,136.14,135.45,134.06,133.65,133.62,131.61,129.85,129.66$, $129.12,128.84,128.73,127.68,127.40,126.90,126.78,122.44,120.40$, $119.79,111.90,103.03,21.42,20.99$.

3-(1-(p-Bromophenyl)-4, 5-diphenyl-1H-imidazol-2-yl)-2-(p-tolyl)-1Hindole $(4 \mathrm{~h})$

Yield: $48 \%$ as a yellow solid.

mp: $268{ }^{\circ} \mathrm{C}$;

IR (neat): $3065,1490,1388,1242,1068 \mathrm{~cm}^{-1}$;

HRMS (ES $)$ calcd. for $\mathrm{C}_{36} \mathrm{H}_{27} \mathrm{BrN}_{3}[\mathrm{M}+\mathrm{H}]^{+} 580.1388$ Found: 580.1400 ; $\mathrm{m} / \mathrm{z}(\%): 580.1400\left([\mathrm{M}+\mathrm{H}]^{+} 100 \%\right), 582.1371(100 \%)$

${ }^{1} \mathrm{H}$ NMR $\left(500 \mathrm{MHz}, \mathrm{DMSO}-d_{6}\right) \delta: 11.57(\mathrm{~s}, 1 \mathrm{H}), 7.60-7.51(\mathrm{~m}, 3 \mathrm{H}), 7.39$ $(\mathrm{d}, J=7.9 \mathrm{~Hz}, 1 \mathrm{H}), 7.34-7.14(\mathrm{~m}, 13 \mathrm{H}), 7.09-7.02(\mathrm{~m}, 3 \mathrm{H}), 6.44(\mathrm{~d}, J=5.5$ $\mathrm{Hz}, 2 \mathrm{H}), 2.31(\mathrm{~s}, 3 \mathrm{H})$.

${ }^{13} \mathrm{C}$ NMR (126 MHz, DMSO- $d$ ) $\delta: 143.08,138.19,138.11,137.85$ $136.34,135.83,135.27,131.64,131.46,131.22,129.99,129.80,129.70$, $129.61,129.23,129.13,128.97,128.75,128.65,128.50,127.60,127.00$ $122.65,121.02,120.51,119.86,112.05,102.56,21.37$.

3-(1-(p-Fluorophenyl)-4,5-diphenyl-1H-imidazol-2-yl)-2-( $p$-tolyl)-1Hindole (4i)

Yield: $54 \%$ as a yellow solid.

mp: $248-250{ }^{\circ} \mathrm{C}$;

IR (neat): $3186,1600,1510,1223,1051 \mathrm{~cm}^{-1}$;

HRMS (ES $)$ calcd. for $\mathrm{C}_{36} \mathrm{H}_{27} \mathrm{FN}_{3}[\mathrm{M}+\mathrm{H}]^{+} 520.2189$ Found: 520.2169 ;

${ }^{1} \mathrm{H}$ NMR (500 MHz, DMSO-d ) $\delta: 11.54(\mathrm{~s}, 1 \mathrm{H}), 7.60-7.51(\mathrm{~m}, 3 \mathrm{H}), 7.38$ $(\mathrm{d}, J=7.9 \mathrm{~Hz}, 1 \mathrm{H}), 7.33-7.24(\mathrm{~m}, 5 \mathrm{H}), 7.23-7.12(\mathrm{~m}, 8 \mathrm{H}), 7.07-7.01(\mathrm{~m}$, $1 \mathrm{H}), 6.76-6.68(\mathrm{~m}, 2 \mathrm{H}), 6.54(\mathrm{bs}, 2 \mathrm{H}), 2.31(\mathrm{~s}, 3 \mathrm{H})$

${ }^{13} \mathrm{C}$ NMR (75 MHz, DMSO- $\left.d_{6}\right) \delta: 162.12,158.88,142.56,137.52,137.44$ $136.95,135.64,134.67,132.20,132.17,130.99,130.67,129.62,129.24$ $129.10,128.97,128.55,128.43,128.27,128.13,128.00,127.81,127.04$, $126.91,126.30,121.96,119.82,119.23,114.88,114.58,111.31,101.96,20.78$.

3-(1-(p-Methoxyphenyl)-4,5-diphenyl-1H-imidazol-2-yl)-2-(p-tolyl)$1 \mathrm{H}$-indole $(4 \mathrm{j})$

Yield: $46 \%$ as a white solid.

m.p: $260{ }^{\circ} \mathrm{C}$;

HRMS $\left(\mathrm{ES}^{+}\right)$calcd. for $\mathrm{C}_{37} \mathrm{H}_{30} \mathrm{~N}_{3} \mathrm{O}[\mathrm{M}+\mathrm{H}]^{+}$532.2389 Found: 532.2380;

${ }^{1} \mathrm{H}$ NMR $(500 \mathrm{MHz}$, Acetone-d $) \delta: 10.53(\mathrm{~s}, 1 \mathrm{H}), 7.70-7.63(\mathrm{~m}, 3 \mathrm{H})$ $7.39(\mathrm{~d}, J=8.1 \mathrm{~Hz}, 1 \mathrm{H}), 7.30-7.20(\mathrm{~m}, 9 \mathrm{H}), 7.19-7.05(\mathrm{~m}, 5 \mathrm{H}), 6.47$ (d, $J=$ $7.8 \mathrm{~Hz}, 2 \mathrm{H}), 6.37(\mathrm{~d}, J=7.9 \mathrm{~Hz}, 2 \mathrm{H}), 3.58(\mathrm{~s}, 3 \mathrm{H}), 2.32(\mathrm{~s}, 3 \mathrm{H})$.

${ }^{13} \mathrm{C}$ NMR (75 MHz, DMSO- $d$ ) $\delta: 157.87,142.67,137.39,137.28,136.73$, $135.52,134.85,130.95,129.97,129.18,129.07,129.06,128.94,128.69$, $128.48,128.44,128.32,128.09,128.05,127.98,127.79,127.02,126.78$ $126.23,126.13,121.83,119.72,119.13,113.06,11.22,102.41,54.97,20.75$.

Urease assay
This assay was modified from Berthelot assay and was employed for the determination of urease activity ${ }^{27}$.

Principle Hydrolysis of urea is catalyzed by urease enzyme as under:-

$\left(\mathrm{NH}_{2}\right)_{2} \mathrm{CO}+\mathrm{H}_{2} \mathrm{O} \rightarrow \mathrm{CO}_{2}+2 \mathrm{NH}_{3}$

This reaction of urease is stopped when the phenol-hypochlorite is added to the reaction mixture. It gives a light blue colored complex between ammonia released and phenol-hypochlorite

\section{METHOD}

A total volume of $85 \mu 1$ assay mixture contained $10 \mu 1$ of phosphate buffer of $\mathrm{pH} 7.0$ in each well in the 96-well plate followed by the addition of $10 \mu \mathrm{l}$ of sample solution and $25 \mu \mathrm{l}$ of enzyme solution ( 0.015 units). The contents were pre-incubated at $37^{\circ} \mathrm{C}$ for $10 \mathrm{~min}$. Then, $40 \mu \mathrm{l}$ of urea stock solution $(20 \mathrm{mM})$ was added to each well and incubation continued at $37^{\circ} \mathrm{C}$ for further $10 \mathrm{~min}$ and take pre-read at $625 \mathrm{~nm}$. After taking pre-read at $625 \mathrm{~nm}$ using the 96 -well plate reader Synergy HT, $115 \mu$ phenol hypochlorite reagent was added in each well (freshly prepared by mixing $45 \mu 1$ phenol reagent with $70 \mu 1$ of alkali reagent). For colour development, incubation was done at $37^{\circ} \mathrm{C}$ for another 10 min. Absorbance (after read) was again measured at $625 \mathrm{~nm}$ using the 96 -well plate reader Synergy HT. The percentage enzyme inhibition was calculated by the following formula:

Inhibition $(\%)=100-[($ Abs. of test sample / Abs. of control $) \times \mathbf{1 0 0}]$

$\mathrm{IC}_{50}$ values (concentration at which $50 \%$ enzyme catalyzed reaction occurs) of active compounds were determined by measuring activities at lower concentrations

$(0.5,0.25,0.125,0.06,0.03 \mathrm{mM})$ and data was computed by using EZ-Fit Enzyme

\section{Antioxidant assay}

Antioxidant potential of all the derivatives was checked by the following method.

DPPH radical scavenging activity

The stable 1, 1-diphenyl-2-picrylhydrazyl radical (DPPH) was used for the determination of antioxidant activity according to a reported method ${ }^{28}$. Different concentrations of compounds in respective solvents were added at an equal volume $(10 \mu \mathrm{l})$ to $90 \mu \mathrm{l}$ of $100 \mu \mathrm{M}$ methanolic DPPH in a total volume of $100 \mu 1$ in 96-well plates. The contents were mixed and incubated at $37{ }^{\circ} \mathrm{C}$ for 30 minutes. The absorbance was measured at $517 \mathrm{~nm}$ using Synergy HT BioTek ${ }^{\circledR}$ USA microplate reader. Quercetin and L-ascorbic acid were used as standard antioxidants. The experiments were carried out in triplicates. $\mathrm{IC}_{50}$ values were calculated using EZ-Fit5 Perrella Scientific Inc. Amherst USA software. The decrease in absorbance indicates increased radical scavenging activity which was determined by the following formula.

Percent scavenging activity $=[100-($ Abs of test compound $/$ Abs of control) $\times 100$

All the measurements were done in triplicate and statistical analysis was performed by Microsoft Excel 2003. Results are presented as mean $\pm \mathrm{sem}$.

\section{ACKNOWLEDGEMENTS}

The authors are thankful to Higher Education Commission of Pakistan (HEC) for the award of IRSIP fellowship. M. A. Khan puts on record his thanks for the generous support of HEC through their Analytical support provisions. Prof. Gary S. Molander of Department of Chemistry, University of Pennsylvania, USA, was gracious enough to provide necessary laboratory and other analytical facilities to Sadia Naureen and deserves our thanks.

\section{REFERENCES}

1- J.L. Stanton, M.H. Ackerman, J. Med. Chem. 26, 986-989, (1983)

2- M.P. Leze, A.Palusczak , R. W. Hartmann, M.L. Borgne, Bioorg Med Chem Lett. 18, 4713-4715, (2008)

3- L.Kumar, S. Bala, K, Jeet, Int J Pharm Pharm Sci. 2, 3-33, (2012)

4- Y.M.Al-Hiari, A.M. Qaisi, M.M.Ei-Abadelah, W.Voelter, Monatsh Chem. 137, 243-248, (2006)

5- Y.M. Na, Bull Korean Chem Soc. 31, 3467-3470, (2010)

6- K.C.S. Achar, K.M. Hosamani, H.R. Seetharamareddy, Eur J Med Chem. 45, 2048-2054, (2010)

7- Jawaharmal, H.S. Lamba, S.Narwal, G.Singh, D.R.Saini, A. Kaur, S. Narwal, IGPS. 2, 147-156, (2012)

8- D.R. Robertson, E.E. Beedle, ¡J. Med. Chem. 36, 939-943, (1987)

9- H.M. Refaat ,Eur J Med Chem. 45,2949-2956, (2010)

10- R.E. Boyd, J.B. Press, C.R. Rasmussen, R.B. Raffa, E.E. Codd, C.D.V, 
D.J. Bennett, A.L. Kirifides, J.F. Gardocki, B. Reynolds, J.T. Hortenstein, A.B. Reitz, JJ. Med. Chem. 44, 863-872, (2001)

11- D. Sharma, B. Narasimhan, P. Kumar, V. Judge, R. Narang, E. De Clercq, J. Balzarini, Eur J Med Chem. 44, 2347-2353, (2009)

12- P.W Manley., N.M. Allanson, R.F.G. Booth, P.E. Buckle, E.D. Kunzair, N. Lad, S.M.F. Lai, D.O. Lunt, P.T David,'J. Med. Chem. 30, 1588-1595, (1987)

13- A. Bhatnagar, P.K. Sharma., N. Kumar, Int J Pharmtech Res. 3, 268-282, (2011)

14- S.Naureen, S. Noreen, A.Nazeer, M. Ashraf, U. Alam, M.A.Munawar, M.A.Khan. Med Chem Res. 24(4), 1584-1595, (2015)

15- S. Naureen, F. Chaudhry, N.Asif, A, M.A. Munawar, M.Ashraf, F.H.Nasim, H. Arshad, and M. A.Khan, Eur J Med Chem. 102, 464e470, (2015)

16- L.S.B.Upadhyay, Indian J. Biotechnol. 11: 381-388, (2012)

17- B. B.Sokmen, U.Serpil, H Y.Sarikaya, H.I.Ugras, and R.Yanardag, Appl Biochem Biotechnol. 171, 2030-2039, (2013)
18- M. Hanif, K. Shoaib, M. Saleem,et al,, ISRN Pharm. 2012,9 pages ,(2012) doi:10.5402/2012/928901.

19- O.U.R. Abid, T. M.Babar, F. I .Ali, S.Ahmad, A. Wadood, N. H. Rama, R. Uddin, A. Khan, M.I. Choudhary, ACS Med. Chem. Lett. 1, 145-149, (2010)

20- L.G. Bundy, J M.Bremner, Soil Biol Biochem. 5, 847-853, (1973)

21- M.A.K.Tanoli, Z. Khan, Z. T. Maqsood, L. Iqbal, Mehr Lateef, Z. Hussain, T. Kamal, Middle East J Sci Res. 22 (5), 698-703, (2014)

22- N.Naik ,H.V. Kumar, J.Rangaswamy, S.T. Harim, T.C. Umeshkumar, J App Pharm Sci . 2, 67-74, (2012)

23- V. Sharma, P. Kumar and D. Pathak, J Heterocycl Chem. 47, 491,(2010)

24- J.S.Biradar, P. Rajesab, S.B.Somappa, J.CHEM. 579612,1-8, (2014)

25- B. Robinson, Chem. Rev. 63,373-401, (1963)

26- G. Jones, S. P.Stanforth, The Vilsmeier reaction of non-aromatic compounds. Organic Reactions. 355-686, 2004

27- M.W. Weatherburn, Anal. Chem. 39, 971-973, (1967)

28- G. C. Yen. and D.Y. Chuang, J Microbiol Biotechnol Food Sci. 48, 2760$2765,(2000)$ 